\title{
Magnesium-Gehalt innerer Organe
}

\author{
Magnesium Content in Human Tissues
}

\author{
H. Kollmeier', G. Rothe', Ch. Witting ${ }^{3}$, J. Seemann! P. Wittig' \\ 'Bundesanstalt fūr Arbeitsschutz, Dortmund \\ 2 Hauptverband der gewerblichen Berufsgenossenschaften, St. Augustin \\ ${ }^{3}$ Pathologisches Institut der Universitäthnstitut für Pathologie am Clemenshospital, Münster
}

\begin{abstract}
Zusammenfassung:
An 58 unselektierten Obduktionen wurde mittels flammenloser Atomabsorptionsspektrometria der geivibliche Magnesium-Gehalt des Herzens $(\bar{x} \pm s=729 \pm 205 \mu \mathrm{g} / \mathrm{g} \mathrm{Tg} .130 \pm 8,4 \mu \mathrm{mol} / \mathrm{g} \mathrm{Tg}$.$) . der Niere (589 \pm 110 \mu \mathrm{g} / \mathrm{g}$

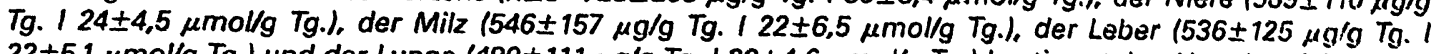

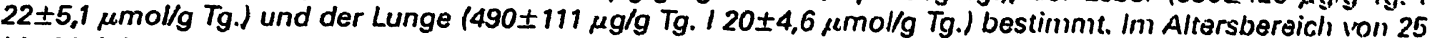
bis 92 Jahren waren die Magnesium-Gehalte in den Organen weder alters-noch geschlechtsabliängig. Es bestand eine hohe Korrelation zwischen dem Gehalt von Magnesium und Zink in Lunge, Leber und Milz sowie zwischen dem von Blei, Magnesium und Zink in Leber. Bei thromboembolischen Prozessen fanden sich niedrige kardiale Magnesium-Gehalte, bei Cholelithiasis und Nephrolithiasis hohe; bei chronischer EmphysemBronchitis waren die Magnesium-Gehalte des Lebergewebes hoch. Im übrigen waren infolge der vielfältigen Multimorbidität des Einzelfalles relativ krankheitshomogene Gruppen kaum abzubilden; desu@gen ist oin Großteil der Ergebnisse kasuistisch dargestellt.
\end{abstract}

\section{Schlüsselwörter:}

Geweblicher Magnesium-Gehalt - Korrelation zwischen Gewebegehalten an Mg, Zn und Pb - Gruppen- und kasuistische Befunde - Atomabsorptionsspektrometrie.

\begin{abstract}
Summary:
In 58 random autopsies, magnesium $(\mathrm{Mg})$ in specimens of heart $(\bar{x} \pm s=729 \pm 205 \mu \mathrm{g} / \mathrm{g} \mathrm{d}$. w. $/ 30 \pm 8.4 \mu \mathrm{mol} / \mathrm{g}$

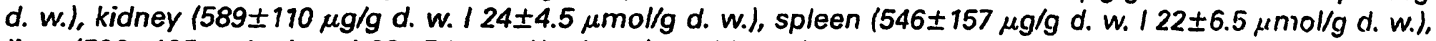

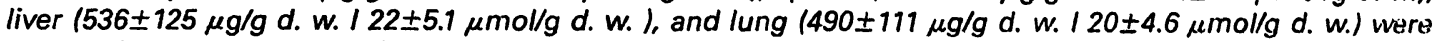
analysed by means of flameless atomic absorption spectrometry. In the examined range from 25 to 92 of age, the $\mathrm{Mg}$ content in all tissues measured did not show age- or sex-dependency. There was a high correlation between the tissue content of $\mathrm{Mg}$ and $\mathrm{Zn}$ in lung, liver and spleen as well as between $\mathrm{Pb}, \mathrm{Mg}$, and $\mathrm{Zn}$ in liver. The $\mathrm{Mg}$ content in heart tissue was low in cases having suffered upon venous and arterial thrombosis and embolism, e. g. pulmonary thromboembolism, ischemic cerebrovascular disease etc.; it was high in cases with cholelithiasis and nephrolithiasis. Chronic bronchitis and emphysema often showed a high $\mathrm{Mg}$ content in liver tissue. Due to the varied multimorbidity of the autopsies examined, it was mostly impossible to build homogenous groups. Thus, a lot of the results has to be demunstrated in a casuistic manner.
\end{abstract}

\section{Keywords:}

Magnesium content in human tissues - correlation between tissue contents of $\mathrm{Mg}, \mathrm{Zn}$, and $\mathrm{Pb}$ - group and casuistic findings - atomic absorption spectrometry.

\section{Einleitung:}

Die Relevanz der Magnesiumionen $\left(\mathrm{Mg}^{++}\right)$für physiologische und pathophysiologische funktionsabläufe findet zunehmende Beachtung, insbesondere ihre Wirkungen am Kreislauf, regulative Effekte auf die Kontraktilität des Herzmuskels und den Tonus der glatten Gefäßmuskulatur, die Modulation der kardialen Erregungsbildung und -leitung, Enzymaktivierungen u. a. Vermutet werden Zusammenhänge zwischen einem chronischen $\mathrm{Mg}^{++}$-Defizit und der Entwicklung eines Hypertonus und einer koronaren Herzkrankheit. Magnesiummangel ist ein wichtiger Intermediärfaktor vielfältiger Nebenwirkungen von Herzglykosiden, Zytostatika, Immunsuppressiva und Antibiotika (9).

Während die $\mathrm{Mg}^{++}$-Bestimmung in Körperflüssigkeiten (2) zur klinischen Routine gehört, sind solche Bestimmun- gen in Organgeweben (3) noch relativ selten, zum Teil auch noch zu optimieren. Der $\mathrm{Mg}^{+4}$-Gehalt in den Erythrozyten und im Knochen wurde als Maß des Körpergehaltes an diesem Kation herangezogen. Wir haben in einer Meßserie den Gehalt an $\mathrm{Cr}, \mathrm{Ni}, \mathrm{Cd}, \mathrm{Pb}, \mathrm{Zn}$ und $\mathrm{Mg}$ in Herz-, Lunge-, Leber-, Milz- und Nierengewebe bestimmt (4): Die dabei gewonnenen Mg-Gehalte werden nachfolgend mitgeteilt.

\section{Material und Methoden}

Untersucht wurden Gewebeproben von 58 unselektierten Obduktionen des Pathologischen Institutes der Universität Münster. Bis auf Einzelfälle, in denen dies aus obduktionsspezifischen Gründen nicht möglich war, wurden die Gewebeproben jeweils aus gleichen Organregionen entnommen: das Herzgewebe vom linken Ventrikel, das Lungengewebe aus den lateralen basalen Partien des rechten

$$
\text { .. Lab.med. 14: } 121 \text { (1990) }
$$


Oberlappens, das Lebergewebe aus der lateralen Vorderfläche, das Milz. gewebe aus der mittleren Vorderflä. che und das Nierengewebe aus dem dorsalen Bereich des unteren Pols (vorwiegend Rinde, aber auch Mark). Die an verschiedenen Todesursachen Verstorbenen waren 25-92 Jahre alt geworden.

Für die Mg-Messung wurde das Gewebe nach Gefriertrocknung unter Druck mit $\mathrm{HNO}_{3}, \mathrm{H}_{2} \mathrm{SO}_{4}$ und $\mathrm{HF}$ aufgeschlossen.

Nach dem Abrauchen, Einstellen auf $\mathrm{pH}$ 2-3 und Verdünnen wurde mittels flammenloser Atomabsorptionsspektrometrie (Perkin-Elmer 5000) bei $202,5 \mathrm{~nm}$ gemessen. Die Eichung erfolgte durch Additionsanalyse. Die Bestimmung der übrigen Metalle ist an anderer Stelle beschrieben (8).

Die analytischen Kenndaten sind:

- Nachweisgrenze (definiert als dreifache Standardabweichung des Leerwertes): $20 \mu \mathrm{g} / \mathrm{g} \mathrm{Tg.;}$

- Präzision ( $n=9):$ VK $=3 \%$;

- Richtigkeit (überprüft mit NBS SRM 1577 bovine liver) ( $n=9$ ): $595 \pm 16 \mu \mathrm{g} / \mathrm{g} \mathrm{Tg}$. gegenüber $604 \pm 9 \mu \mathrm{g} / \mathrm{g} \mathrm{Tg}$. des Referenzmaterials.

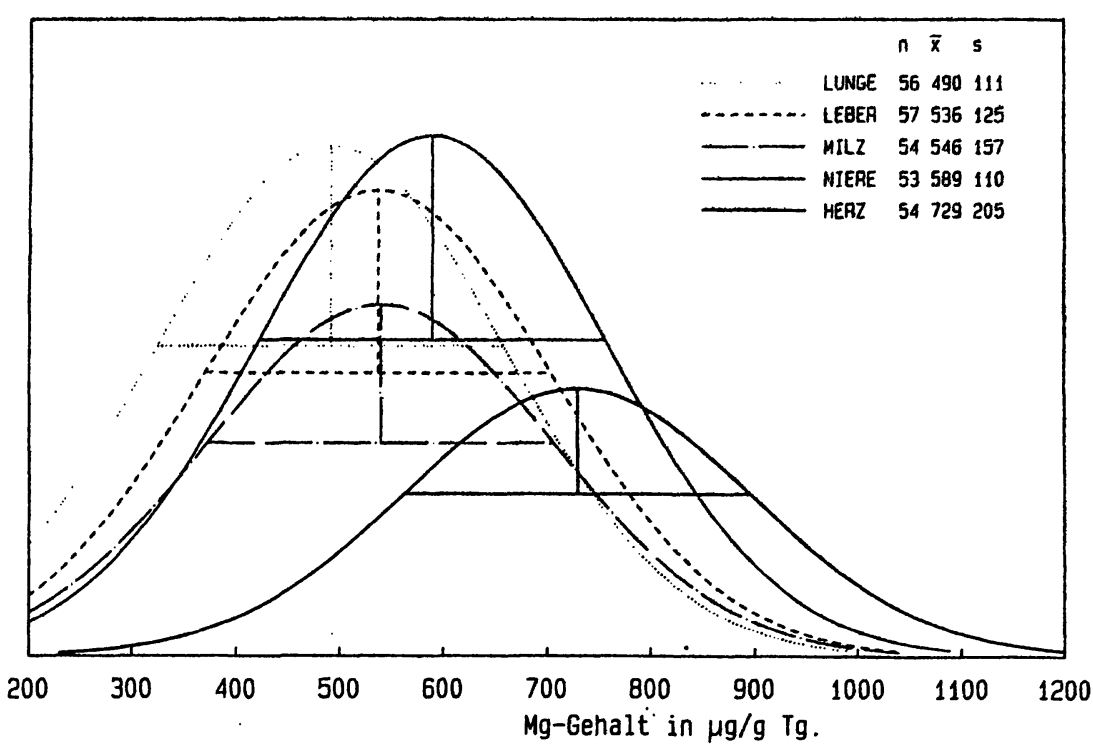

Abb. 1: Mitte/werte und Standardabweichungen der Mg-Gehalte in Gewebeproben von Lunge, Leber, Milz, Niere und Herz: weitgehend normal verteilt (nach Elimination des Extremfalles Nr. 29: s. Abb. 2).

Eine Korrektur der Mg-Meßwerte im Hinblick auf den unterschiedlichen Blutgehalt der Organe wurde (auch wegen der diesbezüglich großen interindividuellen Variabilität in der Meßserie) nicht durchgeführt.

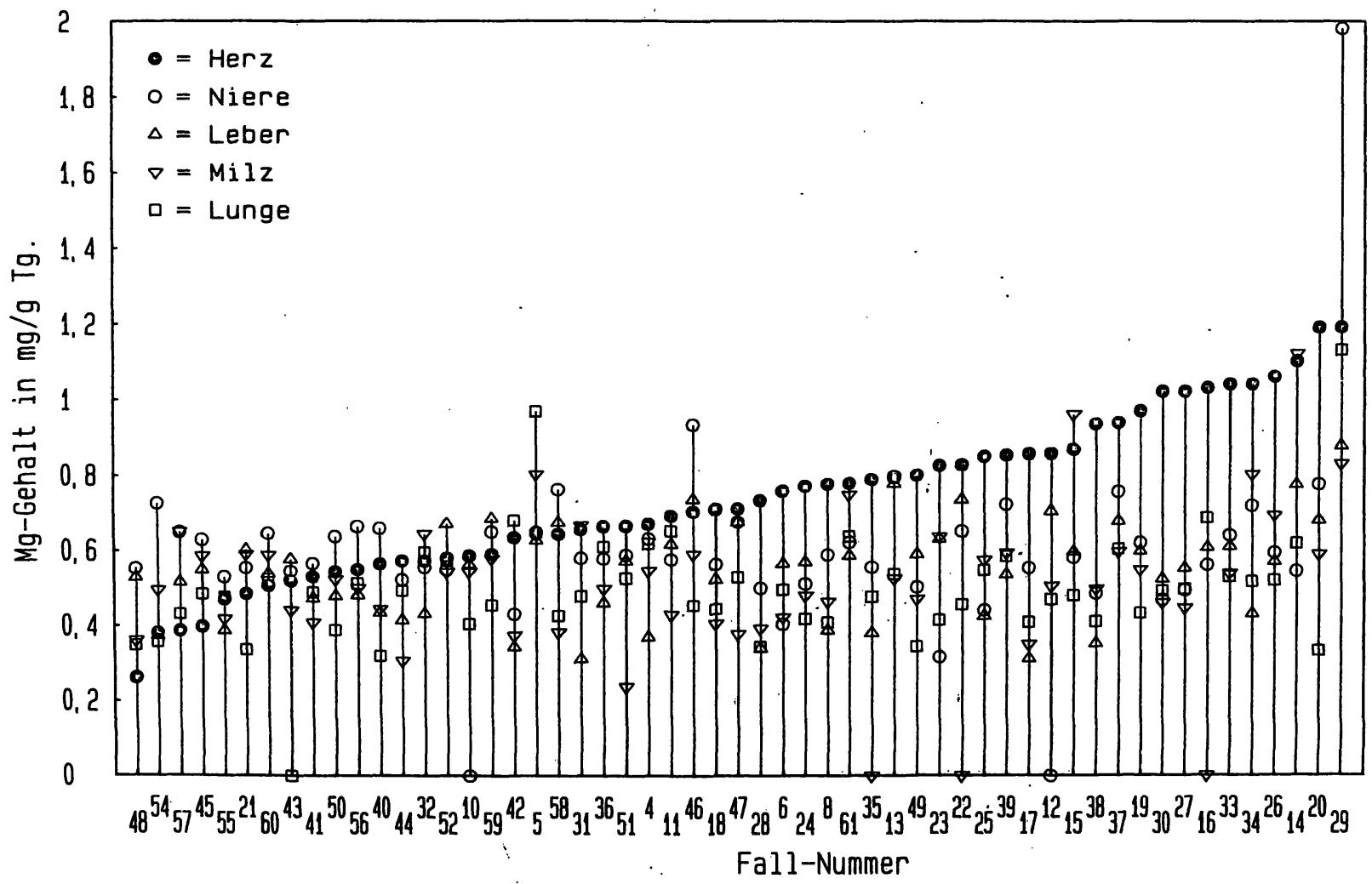

Abb. 2: Kasuistische Darstellung der geweblichen'Mg-Gehalte in Herz, Niere; Leber, Milz und Lunge, geordnet nach dem Anstieg des kardialen Mg-Gehaltes (Fall-Nrn. wie in Tab. 1); die einzelnen Markierungen auf der Grundlinie bedeuten, daß die entsprechenden Organgewebe nicht gemessen werden konnten. 
Tab. 1: $\mathrm{Mg}^{++}$-Gehalte in $\mu \mathrm{g} / \mathrm{g} \mathrm{Tg}$. des Herzens (He), der Niere (Ni) und der Leber (Le) und autoptische Hauptdiagnosen, Kasuistik des auffälligeren Teils der Untersuchung.

\begin{tabular}{|c|c|c|c|c|c|}
\hline Nr. & Alter & He & $\mathbf{N i}$ & Le & Hauptkrankheiten, "Todesursache \\
\hline 48 & 73 & 261 & 552 & 528 & $\begin{array}{l}\text { Arteriosklerose, chron. Linksherzinsuffizienz, Cor pulmonale, thrombotischer Verschluß der } \\
\text { A. car. int. *Hirninfarkt, zentraler Tod } 8 \text { Tage nach Klinikaufnahme. }\end{array}$ \\
\hline 54 & 39 & 381 & 725 & 373 & Ruptur eines intracerebralen Angioms. *Ventrikeltamponade. \\
\hline 57 & 76 & 386 & 648 & 515 & Posthepatitische Leberzirrhose. *Leberkoma. \\
\hline 45 & 81 & 397 & 628 & 548 & $\begin{array}{l}\text { Hypertonie, Arteriosklerose, Herzgewicht } 450 \mathrm{~g} \text {, Wandstärke li } 2 \mathrm{~cm} \text {, Erweichungsherde der } \\
\text { Stammganglien, Hemiparese, Lungeninfarkte bds. *Protrahierte Lungenembolie. }\end{array}$ \\
\hline 55 & 69 &. .470 & 529 & 387 & $\begin{array}{l}\text { Kombiniertes Aortenvitium infolge Endokarditis, chron. Linksherzinsuffizienz, thromboem- } \\
\text { bolischer Verschluß der A. il. re, Beinvenenthrombose. *Rezidivierende Lungenembolie. }\end{array}$ \\
\hline 43 & 84 & 519 & 544 & 577 & $\begin{array}{l}\text { Arteriosklerose, mehrere cerebrale Insulte und Massenblutung, chronisch-obstruktive Lungen- } \\
\text { erkrankung, globale Herzinsuffizienz, Wandstärke li } 1,7 \mathrm{~cm}, \text { re } 0,7 \mathrm{~cm} \text {. *Kardio-respiratorische } \\
\text { Insuffizienz. }\end{array}$ \\
\hline 50 & 78 & 543 & 637 & 479 & $\begin{array}{l}\text { Arteriosklerose, chron. Linksherzinsuffizienz, offenes Foramen ovale, multiple Organinfarkte, } \\
\text { Beinvenenthrombose. *Darminfarkt. }\end{array}$ \\
\hline 56 & 26 & 549 & 664 & 482 & $\begin{array}{l}\alpha_{1} \text {-Antitrypsinmangel (MZ-Typ), Leberzirrhose, thrombosierte Oesophagusvarizen, obere gastro- } \\
\text { intestinale Blutung. *Protrahierter Schock. }\end{array}$ \\
\hline 44 & 74 & 572 & 522 & 415 & $\begin{array}{l}\text { Endokarditis der Mitral- und Aortenklappe, rezidivierte periphere Embolien (Gehirn, Milz, Nieren } \\
\text { und Darmarterien). *Protrahierter Schock. }\end{array}$ \\
\hline 59 & 69 & 590 & 650 & 685 & $\begin{array}{l}\text { Arteriosklerose, multiple Myokard- und Hirninfarkte, Aortenaneurysma mit Zustand nach Ruptur } \\
\text { und nach Implantation einer Y-Prothese. *Globales Herzversagen. }\end{array}$ \\
\hline 5 & 45 & 643 & 649 & 627 & $\begin{array}{l}\text { Metasystox-Intoxikation (Suizid), anfangs therapeutische Stabilisierung, } 2 \text { Wochen später } \\
\text { Magenresektion wegen massiver intestinaler Blutung. *Tags später Tod im prothrahierten } \\
\text { Schock, Schocklungen. }\end{array}$ \\
\hline 58 & 62 & 643 & 762 & 674 & $\begin{array}{l}\text { Hemikolektomie wegen Adenokarzinoms des Kolons (Lebermetastase), nachfolgende Naht- } \\
\text { dehiszenz, Peritonitis, chron. Bronchitis, Bronchiektasen, Emphysem, *Protrahierter Schock. }\end{array}$ \\
\hline 51 & 54 & 664 & 587 & 571 & $\begin{array}{l}\text { Kombiniertes Aorten- und Mitralvitium, Aneurysma dissecans der Aorta, Doppelklappenersatz } \\
\text { und gleichzeitige Operation des Aneurysmas, Herzschrittmacher. *Kardiogener Schock. }\end{array}$ \\
\hline 11 & 74 & 691 & 575 & 616 & $\begin{array}{l}\text { Chronisch-obstruktive Lungenerkrankung, metastasierendes Bronchialkarzinom. Lymphknoten- } \\
\text { metastasen, Arteriosklerose, globale Herzinsuffizienz. *Retentionspneumonie, kardio-respirato- } \\
\text { rische Insuffizienz. }\end{array}$ \\
\hline 46 & 83 & 702 & 933 & 735 & $\begin{array}{l}\text { Oberschenkelamputation wegen feuchter Gangrän, alter Hinterwandinfarkt, chron. Linksherz- } \\
\text { insuffizienz, Rechtsherzüberlastung, chronisch-obstruktive Lungenerkrankung. } \\
\text { * Tod tags nach Amputation infolge kardio-respiratorischer Insuffizienz. }\end{array}$ \\
\hline 24 & 70 & 770 & 510 & 568 & $\begin{array}{l}\text { Leberzirrhose, Alkoholabusus, Arteriosklerose, alter Herzinfarkt, Kardiomegalie, Wand- } \\
\text { stärke li } 1,6 \mathrm{~cm} \text {. *Rezidivierende Hirnblutung. }\end{array}$ \\
\hline 23 & 66 & 825 & 315 & 630 & $\begin{array}{l}\text { Metastasierendes (Hiluslymphknoten, Pleura, Herzbeutel, Leber, Nebenniere) Bronchialkarzinom, } \\
\text { hypernephroides Karzinom, Tumorkachexie. *Retentionspneumonie. }\end{array}$ \\
\hline 39 & 72 & 852 & 721 & 534 & $\begin{array}{l}\text { Rezidivstruma, Strumektomien einen Monat vor dem Tode und fünf Jahre vorher, Cholelithiasis, } \\
\text { Beinvenenthrombose, alte Lungeninfarkte, Rechtsherzinsuffizienz. *Rezidivierende Lungen- } \\
\text { embolien. }\end{array}$ \\
\hline 15 & 86 & 868 & 580 & 596 & $\begin{array}{l}\text { Hypertonie, ausgeprägte Arteriosklerose, rezidivierte Herzinfarkte, Wandstärke li } 1,8 \mathrm{~cm} \text {, } \\
\text { Nephrolithiasis, Cholelithiasis. *Rezidivierter Herzinfarkt mit frischen Herzmuskelnekrosen. }\end{array}$ \\
\hline 19 & 55 & 969 & 619 & 597 & $\begin{array}{l}\text { Adipositas, Diabetes mellitus, chron. Pyelonephritis, Choleithiasis, Gallenblasenperforation. } \\
\text { *Peritonealer Schock. }\end{array}$ \\
\hline 30 & 68 & 1020 & 467 & 522 & $\begin{array}{l}\text { Rezidivierende Urolithiasis, zweimal operiert, Pyelonephritis, Hydronephrose, Myokarditis, } \\
\text { Kardiomegalie, Cholelithiasis. *Herzversagen bei Vorschädigung des Herzens und florider } \\
\text { abszedierender Pyelonephritis. }\end{array}$ \\
\hline 27 & 56 & 1020 & 493 & 551 & $\begin{array}{l}\text { Arteriosklerose, Medianekrosis aortae, Aneurysma dissecans, Wandstärke li } 1,6 \mathrm{~cm} \text {. } \\
\text { *Aortenruptur, Herzbeuteltamponade. }\end{array}$ \\
\hline 16 & 44 & 1030 & 560 & 607 & $\begin{array}{l}\text { Magenkarzinom, totale Gastrektomie und Splenektomie, Relaparotomien wegen subphrenischen } \\
\text { Abszesses bei Nahtinsuffizienz und Pertioneallavage, Bronchopneumonie. *Tod einen Monat } \\
\text { nach Gastrektomie im protrahierten Schock. }\end{array}$ \\
\hline 33 & 72 & 1040 & 639 & $609^{\circ}$ & $\begin{array}{l}\text { Chronisch-obstruktive Lungenerkrankung, Klinikeinweisung wegen peripherer Lungenembolien, } \\
\text { tracheotomiert, maschinell beatmet, Bronchopneumonie, globale Herzinsuffizienz. *Zwei Monate } \\
\text { später Tod infolge kardio-respiratorischer Insuffizienz. }\end{array}$ \\
\hline 34 & 60 & 1040 & 718 & 430 & $\begin{array}{l}\text { Polytrauma, Fettembolie, ausgeprägte Arteriosklerose, fortgeschrittene Leberfibrose. *Protra- } \\
\text { hierter Schock. Schocklungen. }\end{array}$ \\
\hline 26 & 72 & 1060 & 593 & 569 & $\begin{array}{l}\text { Metastasierendes Bronchialkarzinom, Lymphknotenmetastase, chronisch-obstruktive Lungen- } \\
\text { erkrankung, Cor pulmonale, Cholelithiasis, Choledocholithiasis. *Respiratorische Insuffizienz. }\end{array}$ \\
\hline 14 & 57 & 1100 & 543 & 774 & $\begin{array}{l}\text { Luetisches Aortenaneurysma, Operation wegen Perforation, chronisch-obstruktive Lungener- } \\
\text { krankung, Bronchopneumonie. Tod } 8 \text { Tage nach Operation infolge respiratorischer Insuffizienz. }\end{array}$ \\
\hline 20 & 40 & 1190 & 775 & 679 & $\begin{array}{l}\text { Mammakarzinom, Polychemotherapie (6 Serien), Perikarditis carcinomatosa, hämorrhagischer } \\
\text { Perikarderguß. *Kardiogener Schock (3 Jahre nach Ablatio mammae). }\end{array}$ \\
\hline 29 & 25 & 1190 & 1980 & 877 & Anaplastisches metastasiertes Pankreaskopfkarzinom. *Tumorkachexie. \\
\hline
\end{tabular}




\section{Ergebnisse}

Mittelwerte und Streuungen der jeweiligen $\mathrm{Mg}^{++}-\mathrm{Ge}$ halte sind in Abb. 1 dargestellt. In dem randomisierten Obduktionsgut sind die $\mathrm{Mg}^{+4}$-Gehalte der einzelnen Organe, wenn man den Extremfall-Nr. 29 (Abb. 2) ausnimmt, annähernd normalverteilt.

Infolge der vielfältigen Multimorbidität jedes Einzelfalls ist es kaum möglich, relativ krankheitshomogene Gruppen zu bilden. Deswegen ist die individuelle Verteilung der Magnesiumionen auf die großen inneren Organe in Abb. 2 für die gesamte Meßserie wiedergegeben, in Tabelle 1 ergänzt durch Kasuistiken des auffälligeren Teils der Untersuchung. Für die klinische Diskussion können sich einige Hinweise ergeben, gewisse Trends vermeint man zu erkennen:

- überwiegend niedrige $\mathrm{Mg}^{++}$-Gehalte der Herzmuskulatur bei thromboembolischen Prozessen - Lungenembolien u. a., Hirninfarkten u. a. (*Fall-Nrn. 48, 45, $55,43,50,44,59: 479 \pm 116 \mu \mathrm{g} / \mathrm{g} \mathrm{Tg}$. $120 \pm 4,8 \mu \mathrm{mol} / \mathrm{g}$ $\mathrm{Tg.l}$; in diesen Fällen übersteigen fast alle renalen $\mathrm{Mg}^{++}$-Gehalte die kardialen;

- relativ hohe $\mathrm{Mg}^{++}$-Gehalte der Leber bei chronischer Emphysem-Bronchitis $(* 43,58,11,46,33,26,14$ : $651 \pm 79 \mu \mathrm{g} / \mathrm{g} \mathrm{Tg} .127 \pm 3,2 \mu \mathrm{mol} / \mathrm{g}$ Tg. $)$, z. T. verbunden mit auffallend hohen $\mathrm{Mg}^{++}$-Gehalten des Herzens $(* 33,26,14)$;

- hohe renale $\mathrm{Mg}^{++}$-Gehalte bei posthepatitischer ${ }^{*}$ (57) Leberzirrhose und infolge $\alpha_{1}$-Antitrypsinmangels (*56) sowie bei fortgeschrittener Leberfibrose (*34), niedrige bei einer alkoholischen Leberzirrhose $(* 24)$;

- hohe $\mathrm{Mg}^{++}$-Gehalte des Herzens bei Cholelithiasis (*39, 15, 19, 30, 26:954 $\pm 92 \mu \mathrm{g} / \mathrm{g}$ Tg. I 39 $\pm 3,8 \mu \mathrm{mol} / \mathrm{g}$ Tg.) und Nephrolithiasis $(* 15,30)$.

Altersabhängigkeit der $\mathrm{Mg}^{++}$-Gehalte ist nicht nachzuweisen, auch keine Geschlechtsabhängigkeit.

Hinsichtlich der $\mathrm{Mg}^{++}$-Gehalte ergibt sich zwar eine auffällige Spearmansche Rangkorrelation von Leber zu Milz ( $p$-Wert 0,03$)$, zu Niere $(0,01)$ und zu Herz $(0,04)$; es liegt aber keine Signifikanz zum multiplen Niveau unter 0,15 vor, so daß nicht schon angenommen werden kann, diese Beziehungen hielten einer validierten Überprüfung stand.

Gefunden wurde eine hohe Korrelation zwischen den Gehalten von Magnesium und Zink in Lunge $(0,0001)$, Leber $(0,0001)$ und Milz $(0,0008)$ sowie zwischen der von Blei, Magnesium $(0,002)$ und Zink $(0,0003)$ in Leber $(5,6)$.

\section{Diskussion}

Durch Gefriertrocknung vor dem analytischen Gewebeaufschluß und Bezug des $\mathrm{Mg}$-Gehaltes auf $\mathrm{g}$. Tg. wird ein Bestimmungsfehler eliminiert, der beim Aufschluß von Frischproben auftritt, weil der Wassergehalt der Gewebe stark schwanken kann, und zwar sowohl innerhalb eines Organs als auch interindividuell. Dies gilt besonders für Lungengewebe; in einer anderen Meßserie $(n=258)$ variierte hier der Umrechnungsfaktor (Tg./Frischgewicht) zwischen 4,1 und 12,8 bei einer mittleren Varianz (VK) von $16 \%$ (4); bei Formalin-konservierten Proben betrug diese Varianz sogar $20 \%$ (7). Wenn nur jeweils eine Organprobe entnommen wird, ist eine gewisse intraorganische Variabilität der $\mathrm{Mg}^{++}$-Gehalte in Rechnung zu stellen; sie wurde in der vorliegenden Untersuchung nicht genügend geprüft, weil wir anfangs davon ausgingen, daß dieses "physiologische" Element in den Organen weitgehend gleich verteilt sei.

Die $\mathrm{Mg}^{++}$-Gehalte in den einzelnen Organen sind weitgehend normalverteilt. Da die Untersuchung an einem unselektierten Obduktionsgut mit Multimorbidität erfolgte, bleibt offen, ob sich die entsprechenden Normbereiche der jeweiligen organgeweblichen $\mathrm{Mg}^{++}$-Gehalte mit den vorgefundenen Standardstreubereichen decken.

Hinsichtlich der Vielfalt der pathologischen Diagnosen fallen immerhin die niedrigen $\mathrm{Mg}^{++}$-Gehalte der Herzmuskulatur bei thromboembolischen Prozessen (Lungenembolien u. a., Hirninfarkte u. a.) auf. Yamori (10) berichtete über Experimente mit SHRSP-Ratten - die infolge selektiver Zucht neben Hypertonie fast alle einen cerebralen Insult erleiden - , in denen durch $\mathrm{Mg}$-angereicherte Diät eine sehr deutlicher Präventionseffekt erzielt wurde. Diese und analoge klinische und epidemiologische Beobachtungen $(1,9)$ setzen die niedrigen kardialen $\mathrm{Mg}^{++}-\mathrm{Ge}-$ halte zu den (links-)kardialen Insuffizienzen mit ihren Rückwirkungen auf Gefäßtonus und -alteration sowie Blutzirkulation und -gerinnung in eine gewisse Beziehung.

Die Homöostase von Magnesium und Calcium ist oft gleichzeitig gestört; Einflüsse des Parathormons sind nachgewiesen. Seit Jahren wird diskutiert, ob dem Einsatz von Magnesiumsalzen in der Prophylaxe der rezidivierenden Nierensteinkrankheit eine entscheidende Rolle zukommt; die entsprechenden Beobachtungen sind widersprüchlich (1). In diesem Zusammenhang ist bemerkenswert, daß alle Steinleiden der Meßserie (Cholelithiasis und Urolithiasis: Steinanalysen wurden nicht vorgenommen) einen hohen kardialen $\mathrm{Mg}^{++}$-Gehalt aufwiesen.

Schrifttum:

1. BALTZER, G.: Klinische Aspekte des Magnesiumstoffwechsels. Med. Klin. 83, 370-372 (1988)

270-372 (1988). 2. FALCK, M., CEPELNIK, E. M., RICK, W.: Die Bestimm
tion in Körperflüssigkeiten. Internist 30, 51-56 (1989).

3. IYENGAR, G. V., KOLLMER, W. E., BOWEN, H. J. M.: The elemental composition of human tissues and body fluids. Verlag Chemie, Weinheim-New York (1978).

4. KOLLMEIER, H., MÜLLER, K. M., SEEMANN, J., ROTHE, G., WITTIG, P., SCHEJBAL, V. HUMMELSHEIM, G.: Untersuchung zur Chrom- und Nickel-Belastung der Lunge. Wirtschaftsverlag NW, Bremerhaven (BAU Fb 548, S 1-222) (1988).

5. KOLLMEIER, H., WITTING, C., SEEMANN, J., WITTIG, P., ROTHE, G.: Metallanreicherungen in Humangeweben. Chrom, Nickel, Blei, Cadmium und Zink in Lunge, Leber, rungen in Humangewer. (1985). 6. ROTHE, G.: Einige statistische Aspekte bei der Auswertung und Interpretation von Messungen des Gehaltes an bestimmten Metallen $(\mathrm{Cr}, \mathrm{Ni}, \mathrm{Pb}, \mathrm{Cd}, \mathrm{Zn}, \mathrm{Mg}$ ) in Humangeweben. Universität Dortmund (Arbeitsberichte der Abteilung Statistik, Nr. 15) (1984). 7. SEEMANN, J., WITTIG, P., KOLLMEIER, H., MÜLLER, K. M.: Trace metal analysis of chromium and nickel in lung tissue fixed and stored in formalin. Path. Res. Prac. (in press) (1989)

8. SEEMANN, J., WITTIG, P., KOLLMEIER, H., ROTHE, G.: Analytische Bestimmung von $\mathrm{Cd}, \mathrm{Pb}, \mathrm{Zn}, \mathrm{Cr}$ und $\mathrm{Ni}$ in Humangewebe. Lab.med. 9, 294-299 (1985).

9. SPATH, G.: Magnesium in der Kardiologie. WMW 138, 382-415 (1988).

10. YAMORI, Y: Experimental and epidemological studies on the role of magnesium in the patho International Magnesium Symposium, Aug. 8-12, Kyoto/Japan (1988).

Anschrift für die Verfasser:

Dr. H. Kollmeier, Dir. u. Prof. Bundesanstalt für Arbeitsschutz Vogelpothsweg 50-52 4600 Dortmund 1 


\section{VE⿱一兀)}

Wirkstoffa Hepartio-Natrium

\section{Promonta flamburig}

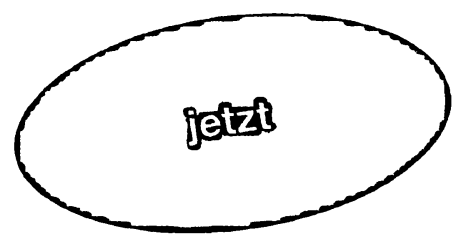

in physiologischer Elektrolytzusammensetzung

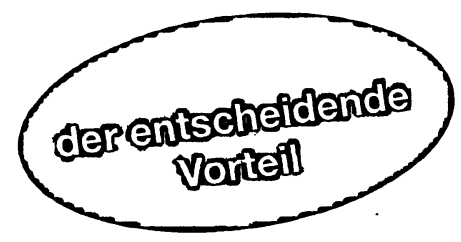

bei der Analytik der Blutgase und/oder Elektrolyte im Vollblut:

Klinisch relevante Fehlbestimmungen können vermieden werden.

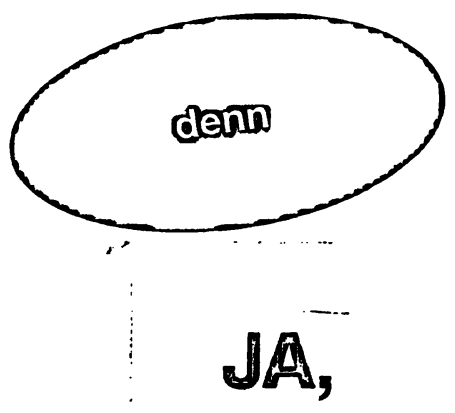

- Durch die minimierte Heparinkonzentration in Vetren $^{\otimes} 200$ wird die Calciumbindung an Heparin umgangen.

-. . . . $ه$ nnn : nt jedoch erhindern.

bitte übersenden Sie mir kostenlos ie ;alcium rkt

\section{Exemplar(e)} „VETREN ${ }^{\circledR} 200$ Gebrauchsinformation zur Vorbereitung von Blutgas-Spritzen" 


\section{Dieses Merkblatt zurProbenvorbereitung mit Vetren ${ }^{\oplus} 200$ übersenden wir Ihnen gern! Fordern Sie es mit der anhängenden Karte*) an!}

\section{VETREN ${ }^{(1)} 200$}

\section{$\circ$}

Gebrauchsinformation zur vorb 200 ist besonders geeignet zur ProbenVETREN 200 für die Analytik der Blulgas. vorbereitung oder der Elektrolys

NON VETREN* Eigenschaften: Die Elektrolytzusammen normalen Blutplasmänus200 ist derjenigen de Weise werden Verdu Spritzengeglichen. Auf diese Wendige Füllung des Sprmie geglich durch die notwendigung weitgehend ver solltotraums mit Heparinlösung wät 4 den.Der Spritzentritze. volumens der Spritze. Die Heparinkonzentr a ngemaßni Die Heparink hoch 9 ţ die Bindung

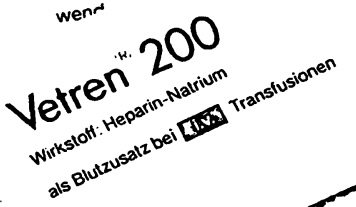
s an Heparin

\section{Promonta Hamburg} "y des ionisier-

$$
\text { B. Probe (ninst an }
$$

\author{
")(Sollte sie bereits \\ fehlen, Postkarte \\ gen(igt!)
}

Zusammensetzung: 1 Ampulle Vetren 200 zu 2 ml enthălt 200 I.E. HeparinNatrium. Anwendungsgebiete: Indirekte Bluttransfusion/Austauschtransfusion bei Neugeborenen, diagnostische Untersuchungen. Gegenanzeigen: Hămorrhagische Diathese, Magen- und Darmgeschwäre Nieren- und Harnleitersteine, Verdacht auf cerarale lschsmien Insult, Operationen an Gehirn, Rockenmark und Augen, drohende Fehlgebur , Pelitien und Plattchenaggregationshemmern (2.B. Acetylsalicylsaure), Heparinallergie, schwere Leber-, Nieren- und Pankreaserkrankungen, Endocarditis lenta, manifeste Hypertonie. Eine Leberblindpunktion sollte wăhrend derZeit einerHeparinTherapie nicht durchgefahrt werden. Menstruationsblutungen stellen keine Kontraindikation dar. (Im Einzelfall sind Nutzen und Risiko der Heparin-Therapie gegeneinander abzuwägen.) Bei manifestem Diabetes ist Vorsicht geboten, da Heparinzufuhr bei diabetischer Stoftwechsellage zu einem vorabergehenden Anstieg der freien Fettsăuren im Blut fähren kann. Die Anwendung von HeparinPrăparaten wăhrend der Schwangerschaft sollte nur nach strenger Abwägung aller Risiken erfolgen. Nebenwirkungen: Gelegentlich Blutungen, die aber nur bei schwerem Verlauf eine Unterbrechung bzw. einen Abbruch der Therapie erfordern In seltenen Fallen überempfindlichkeitsreaktionen, vor allem bie Patienten, die bereits fruher mitHeparin behandelt
Knochenerweichungen mit Neigung zu Brûchen moglich. Ein reversib er Abfall der Thrombozyten ist moglich und kann bei abnehmenden Werten untt $>50000$ eine beginnende Sensibilisierung anzeigen. Ein gelegentlich auftretenc 3 r Haarausfall ist stets vorabergehender Natur und prophylaktisch oder thera, eutisch nicht beeinflußbar. Moglich sind ebenfalls Fieberanstieg. Kopfschmerze I, Übelkeit und Priapismus.

Wechselwirkungen mit anderen Mitteln: Orale Antikoagulantien undTh omboWechselwirkungen mit anderen Mittein: OraleAntikoagulantien undTh ombozytenaggregationshemmer oder andere gerinnungshemmende Stoffe tcetylsalicylsäure, Dicumarol-Derivate, Dipyridamol, Dextrane) können beigle. :Theiti-
ger Anwendung zu einer Wirkungsverstärkung und einem erhohten Bli tungsrisiko führen. Bei gleichzeitiger Gabevon Antihistaminika, Ascorbinsäure, Digitalisglykosiden, Nikotin, Phenothiazinen, Tetrazyklinen und Penicillin kann es zu einer Abschwächung der blutgerinnungshemmenden Wirkung des Heparins kommen.'Hinweise: Zur Neutralisation von intravenठs appliziertem Vetren 200 verabreicht man eine $1 \%$ ige Protaminlosung. 1-1,5 mg Protamin neutralisieren 100 I.E. Heparin, die Injektion kann je nach Schwere des Falles in wenigen Minuten wiederholt werden. Handelsformen: Packung mit 10 Ampullen zu 2 m DM 3,81; Anstaltspackung: 100 Ampullen zu $2 \mathrm{ml} \mathrm{DM} \mathrm{36,22.} \mathrm{(Stand} \mathrm{1/90).}$ 